\title{
ON SIMULTANEOUS EXTENSION OF CONTINUOUS FUNCTIONS
}

\author{
BY H. H. CORSON ${ }^{1}$ AND J. LINDENSTRAUSS ${ }^{2}$
}

Communicated by V. Klee, January 14, 1965

Let $S$ be a compact Hausdorff space and let $K$ be a closed subset of $S$. Denote by $C(S)$ (respectively, $C(K)$ ) the Banach space of all continuous real-valued functions on $S$ (respectively, $K$ ) with the supremum norm. A bounded linear operator $T$ from $C(K)$ to $C(S)$ is called a simultaneous extension (s.e.) operator if the restriction of $T f$ to $K$ is equal to $f$ for every $f \in C(K)$. Put

$$
\eta(K, S)=\inf \{\|T\| ; T \text { is an s.e. operator from } K \text { to } S\} .
$$

$(\eta(K, S)=\infty$ if there exists no s.e. operator from $K$ to $S$.) Several authors (for example, Borsuk, Kakutani, Dugundji and Arens, cf. the expository paper [4] for references) have considered this notion of simultaneous extension of continuous functions. It is known that, if $K$ is metrizable, then $\eta(K, S)=1$ for every $S \supset K$ (cf. the recent paper [3] for a much stronger result), and examples of $K$ and $S$ for which $\eta(K, S)=\infty$ are known. As far as we know, in all examples considered thus far either one of these two extreme situations occurred.

In this note we find all the possible values of $\eta(K, S)$ for $K$ the onepoint compactification of an uncountable set (which is, in a sense, the simplest nonmetrizable compact Hausdorff space). The result we obtain is somewhat surprising and it indicates that the study of the behaviour of $\eta(K, S)$ for more general $K$ may be of interest. We intend to consider this question as well as the more general question of extending maps into nonmetrizable compact convex sets in a future paper (cf. also Proposition 1 in this note).

THEOREM 1. Let $K$ be the one-point compactification of an uncountable set. Then, for every compact Hausdorff $S$ containing $K, \eta(K, S)$ is either an odd integer or $\infty$. Conversely, for every integer $n$ there is an $S_{n} \supset K$ such that $\eta\left(K, S_{n}\right)=2 n+1$ and there is also an $S_{\infty} \supset K$ such that $\eta\left(K, S_{\infty}\right)=\infty$.

Denote by $\Sigma$ the unit cell of $C(K)^{*} . \Sigma$ consists of all measures $\mu$ on $K$ with total variation $\|\mu\| \leqq 1$ and it is compact Hausdorff in

1 Research fellow of the Alfred P. Sloan Foundation.

${ }^{2}$ Research supported in part by the National Science Foundation (NSF GP-378). 
the w*-topology. The point at infinity of $K$ will be denoted by $\infty$ and $K \sim\{\infty\}$ will be denoted by $A$. For $\alpha \in K$ we denote by $\phi_{\alpha}$ the measure which gives mass one to the point $\alpha$ and is zero everywhere else. Every $\mu \in \Sigma$ can be represented as

$$
\mu=\sum_{\alpha \in A} \mu(\alpha) \phi_{\alpha}+\mu(\infty) \phi_{\infty}, \quad\|\mu\|=\sum_{\alpha \in A}|\mu(\alpha)|+|\mu(\infty)| \leqq 1 .
$$

Theorem 1 is an easy consequence of the following two lemmas.

Lemma 1. Let $n$ be an integer and let $2 n-1 \leqq \lambda<2 n+1$. Then there is a $w^{*}$-continuous map $P$ from $\lambda \Sigma$ into $(2 n-1) \Sigma$ such that $P \phi_{\alpha}=\phi_{\alpha}$ for every $\alpha \in K$.

LEMma 2. Let $n$ be an integer and let $\tau<2 n+1$. Then there is no w*continuous map $P$ from $(2 n+1) \Sigma$ into $\tau \Sigma$ such that $P \phi_{\alpha}=\phi_{\alpha}$ for every $\alpha \in K$.

To derive Theorem 1 from these two lemmas we have just to observe that $\eta(K, S) \leqq \lambda$ if and only if there is a continuous map $F$ from $S$ into $\lambda \Sigma$ (in the w*-topology) such that $F(\alpha)=\phi_{\alpha}$ for $\alpha \in K$. Thus, in particular, if $S_{n}=(2 n+1) \Sigma$ and if we consider $K$ as a subset of $S_{n}$ (by identifying $\alpha \in K$ with $\phi_{\alpha}$ ) then, by Lemma $2, \eta\left(K, S_{n}\right)$ $=2 n+1$, and if $S_{\infty}$ is a compactification of $C(K)^{*}$ (with the w*topology) then $\eta\left(K, S_{\infty}\right)=\infty$.

PROOF OF LEMMA 1. Let $\mu=\sum_{\alpha \in A} \mu(\alpha) \phi_{\alpha}+\mu(\infty) \phi_{\infty}$; then $\mu(K)$ $=\sum_{\alpha \in A} \mu(\alpha)+\mu(\infty)$. We take $P=P_{2} P_{1}$, where $P_{1}$ and $P_{2}$ are defined by

and

$$
P_{1}(\mu)=\mu / \max (1, \mu(K))
$$

$$
P_{2}(\mu)=\sum_{\alpha \in A} g(\mu(\alpha)) \phi_{\alpha}+\left(1-\sum_{\alpha \in A} g(\mu(\alpha))\right) \phi_{\infty},
$$

where $g$ is a continuous real-valued function on the line satisfying

$$
0 \leqq g(t) \leqq 1, \quad g(t)=0 \text { if } t \leqq(\lambda+1) /(2 n+2), \quad g(1)=1 .
$$

Both $P_{1}$ and $P_{2}$ are $\mathrm{w}^{*}$-continuous on $\lambda \Sigma$ and $P_{1} \phi_{\alpha}=P_{2} \phi_{\alpha}=\phi_{\alpha}$ for every $\alpha \in K$. If $\mu \in \lambda \Sigma$ and $\gamma=P_{1} \mu$, then $\gamma \in \lambda \Sigma$ and $\gamma(K) \leqq 1$. It follows that there are at most $n$ values of $\alpha$ such that $\gamma(\alpha)>(\lambda+1) /(2 n+2)$ and, hence, $\left\|P_{2} \gamma\right\| \leqq 2 n-1$. This concludes the proof of Lemma 1 .

For the proof of Lemma 2 we need the following simple combinatorial lemma.

Lemma 3. Let $A$ be a uncountable set and let $n$ be an integer. For every $\alpha \in A$ let $\psi(\alpha)$ be a subset of $A$ whose complement is finite. Then there are $\left\{\alpha_{i}\right\}_{i=1}^{n}$ in $A$ such that $\alpha_{i} \in \psi\left(\alpha_{j}\right)$ for every $i \neq j$. 
Proof. By induction on $n$. Assume the lemma holds for some $n \geqq 1$. Pick a $\beta_{1} \in A$. Since $A \sim \psi(\beta)$ is finite for every $\beta$ we can construct a sequence $A_{1} \subset A_{2} \subset A_{3} \subset \cdots$ of finite subsets of $A$ with $A_{1}=\left\{\beta_{1}\right\}$ such that all the inclusions are proper and such that $\beta \in A_{k}$ for some $k$ implies that $A \sim \psi(\beta) \subset A_{k+1}$.

By the induction hypothesis there are $\left\{\alpha_{i}\right\}_{i=1}^{n}$ in the uncountable set $A \sim \bigcup_{k=1}^{\infty} A_{k}$ such that $\alpha_{i} \in \psi\left(\alpha_{j}\right)$ for every $i \neq j$. Since $\bigcap_{i=1}^{n} \psi\left(\alpha_{i}\right)$ has a finite complement and since $\mathrm{U}_{k=1}^{\infty} A_{k}$ is infinite there is an $\alpha_{n+1}$ in $\bigcup_{k=1}^{\infty} A_{k}$ such that $\alpha_{n+1} \in \psi\left(\alpha_{i}\right)$ for $i=1,2, \cdots, n$. The complement of $\psi\left(\alpha_{n+1}\right)$ is contained in $\bigcup_{k=1}^{\infty} A_{k}$ and hence $\alpha_{i} \in \psi\left(\alpha_{n+1}\right)$ for $i=1,2, \cdots, n$. Thus the lemma holds also for $n+1$ and this concludes the proof.

REMARK. Actually it is easy to prove that with the assumptions of Lemma 3 there is an uncountable subset $A^{\prime}$ of $A$ such that $\alpha \in \psi\left(\alpha^{\prime}\right)$ whenever $\alpha, \alpha^{\prime} \in A^{\prime}$ and $\alpha \neq \alpha^{\prime}$. We shall not, however, need this stronger version of the Lemma.

PROOF OF LemMa 2. Let $\tau<2 n+1$ and assume that there is a $\mathrm{w}^{*}$ continuous map $P$ from $(2 n+1) \Sigma$ into $\tau \Sigma$ such that $P \phi_{\alpha}=\phi_{\alpha}$ for every $\alpha \in K$. For $\alpha_{1}, \alpha_{2}, \cdots, \alpha_{n+1} \in A$ put

and

$$
\mu_{\alpha_{1}, \alpha_{2}}, \cdots, \alpha_{n+1}=\phi_{\alpha_{1}}+\phi_{\alpha_{2}}+\cdots+\phi_{\alpha_{n+1}}-n \phi_{\infty},
$$

$$
\gamma_{\alpha_{1}, \alpha_{2}}, \cdots, \alpha_{n+1}=P \mu_{\alpha_{1}, \alpha_{2}}, \cdots, \alpha_{n+1} \text {. }
$$

Let $\epsilon>0$. We claim that for every $\alpha \in A$ there is a subset $\psi(\alpha)$ of $A$ with $A \sim \psi(\alpha)$ finite such that $\beta_{1}, \beta_{2}, \cdots, \beta_{n} \in \psi(\alpha)$ implies that

$$
\gamma_{\alpha, \beta_{1}, \beta_{2}, \cdots, \beta_{n}}(\alpha)>1-\epsilon .
$$

Indeed, if no such $\psi(\alpha)$ exists we would have $\beta_{k}^{j}, j=1,2, \cdots$, $k=1,2, \cdots, n$, in $A$ such that $\beta_{k_{1}}^{f_{1}} \neq \beta_{k_{2}}^{y_{2}}$ if $j_{1} \neq j_{2}$ and

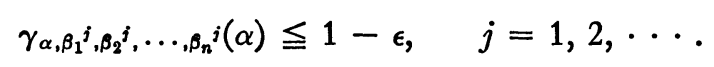

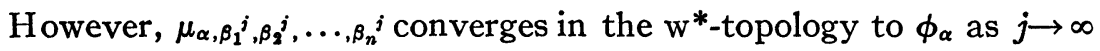

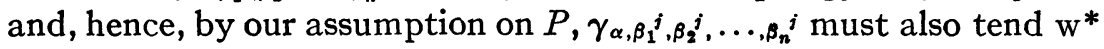
to $\phi_{\alpha}$ and this contradicts (1).

We divide $A$ into a sequence of disjoint uncountable subsets $\left\{A^{j}\right\}_{j=1}^{\infty}$. By Lemma 3 there are, for every $j$, elements $\alpha_{k}^{j} \in A^{j}$, $k=1,2, \cdots, n+1$, such that

$$
\alpha_{k_{1}}^{j} \in \psi\left(\alpha_{k_{2}}^{j}\right) \quad \text { if } \quad k_{1} \neq k_{2}, \quad j=1,2, \cdots
$$

We have thus

(2) $\gamma_{\alpha_{1}{ }^{j}, \alpha_{2}{ }^{j}, \cdots, \alpha_{n+1}^{j}}^{j}\left(\alpha_{k}^{j}\right)>1-\epsilon, k=1,2, \cdots, n+1, j=1,2,3, \cdots$ 
Since $\mu_{\alpha_{1}{ }^{j}, \alpha_{2}^{j}, \cdots, \alpha_{n+1}^{j}}$ converges in the $\mathrm{w}^{*}$ topology to $\phi_{\infty}$ as $j \rightarrow \infty$ we get, by our assumptions on $P$, that, for sufficiently large $j$,

$$
\gamma_{\alpha_{1}{ }^{j}, \alpha_{2}{ }^{j}, \cdots, \alpha_{n+1}^{j}}^{j}(K)<\phi_{\infty}(K)+\epsilon=1+\epsilon .
$$

For a $j$ satisfying (3) we get easily (by using (2)) that

$$
\left\|\gamma_{\alpha_{1}{ }^{j}, \alpha_{2}{ }^{j}, \cdots, \alpha_{n+1}^{j}}\right\| \geqq 2 n+1-(2 n+3) \epsilon,
$$

and this is a contradiction if $\epsilon$ is chosen so that $2 n+1-(2 n+3) \epsilon>\tau$. This concludes the proof of Lemma 2 and, hence, of Theorem 1.

The method of proof of Lemma 2 can be used to prove results concerning mappings into other compact convex sets besides sets of measures. It is known that every compact convex set in a metrizable locally convex space (e.g. the unit cell of a separable reflexive space in the w-topology) is a retract of every compact space containing it (cf. [2] and the references there). It was observed in [1] and [2] that the unit cell of a nonseparable Hilbert space in the w-topology does not have this property, since it can be embedded in the product of uncountable copies of $[0,1]$, which is separable. Here we get the following stronger result.

Proposition 1. Let $U$ be the unit cell of a nonseparable Hilbert space and let $\lambda>\tau \geqq 1$. Then there is no w-continuous map $P$ from $\lambda U$ into $\tau U$ such that $P x=x$ for $x \in U$.

The proof is very similar to that of Lemma 2 . We just have to replace the measures $\mu_{\alpha_{1}}, \cdots, \alpha_{n+1}$ by the points

$$
x_{\alpha_{1}, \alpha_{2}}, \cdots, \alpha_{n}=\lambda\left(e_{\alpha_{1}}+e_{\alpha_{2}}+\cdots+e_{\alpha_{n}}\right) / \sqrt{ } n
$$

in $\lambda U$ (here $n$ is an integer $\geqq \lambda^{2}$ and the $\left\{e_{\alpha}\right\}_{\alpha \in A}$ form an orthonormal basis of the Hilbert space).

Proposition 1 holds also if $U$ is the unit cell of a nonseparable $l_{p}$ space, $1 \leqq p<\infty$, in the $\mathrm{w}^{*}$-topolog $\mathrm{y}$, but it does not hold for the unit cell of $l_{\infty}$ in the $\mathrm{w}^{*}$-topology.

\section{REFERENCES}

1. V. Klee, Convex bodies and periodic homeomorphisms in Hilbert space, Trans. Amer. Math. Soc. 74 (1953), 10-43.

2. E. Michael, Some extension theorems for continuous functions, Pacific J. Math. 3 (1953), 789-806.

3. A. Pelczynski, On simultaneous extension of continuous functions, Studia Math. 24 (1964), 286-304.

4. Z. Semadeni, Isomorphic properties of Banach spaces of continuous functions, Studia Math. Seria. Specjalna (1963), 93-108.

UNIVERSITY OF WASHINGTON 